\title{
Sexual and asexual oogenesis require the expression of unique and shared sets of genes in the insect Acyrthosiphon pisum
}

\author{
Aurore Gallot', Shuji Shigenobu², Tomomi Hashiyama², Stéphanie Jaubert-Possamai ${ }^{1 * \dagger}$ and Denis Tagu ${ }^{1 *+}$
}

\begin{abstract}
Background: Although sexual reproduction is dominant within eukaryotes, asexual reproduction is widespread and has evolved independently as a derived trait in almost all major taxa. How asexuality evolved in sexual organisms is unclear. Aphids, such as Acyrthosiphon pisum, alternate between asexual and sexual reproductive means, as the production of parthenogenetic viviparous females or sexual oviparous females and males varies in response to seasonal photoperiodism. Consequently, sexual and asexual development in aphids can be analyzed simultaneously in genetically identical individuals.

Results: We compared the transcriptomes of aphid embryos in the stages of development during which the trajectory of oogenesis is determined for producing sexual or asexual gametes. This study design aimed at identifying genes involved in the onset of the divergent mechanisms that result in the sexual or asexual phenotype. We detected 33 genes that were differentially transcribed in sexual and asexual embryos. Functional annotation by gene ontology (GO) showed a biological signature of oogenesis, cell cycle regulation, epigenetic regulation and RNA maturation. In situ hybridizations demonstrated that 16 of the differentially-transcribed genes were specifically expressed in germ cells and/or oocytes of asexual and/or sexual ovaries, and therefore may contribute to aphid oogenesis. We categorized these 16 genes by their transcription patterns in the two types of ovaries; they were: i) expressed during sexual and asexual oogenesis; ii) expressed during sexual and asexual oogenesis but with different localizations; or iii) expressed only during sexual or asexual oogenesis.
\end{abstract}

Conclusions: Our results show that asexual and sexual oogenesis in aphids share common genetic programs but diverge by adapting specificities in their respective gene expression profiles in germ cells and oocytes.

\section{Background}

Sexual reproduction involves two main events: meiosis and fertilization, and creates new genotypes by shuffling allelic combinations. Although the predominance of sexual reproduction in eukaryotes supports this innovation as a successful reproduction strategy, asexuality has evolved independently multiple times from sexual ancestors in almost all major taxa [1-4], such as in stick insects [3] and Ranunculus plants [4]. How asexuality has evolved in sexual organisms is unclear. In aphids, asexuality was acquired once about 250 million years

\footnotetext{
* Correspondence: stephanie.jaubert@sophia.inra.fr; denis.tagu@rennes.inra.fr + Contributed equally

'INRA, UMR 1349 IGEPP, Institut de Génétique Environnement et Protection des Plantes, 35653 Le Rheu cedex, France

Full list of author information is available at the end of the article
}

ago by a common sexual ancestor [5]. Most aphid species alternate between sexual reproduction and asexual parthenogenetic reproduction according to seasonal variations. In spring and summer, aphids reproduce asexually by parthenogenesis and produce clonal parthenogenetic female progeny by viviparity. The autumnal shortening of the photoperiod induces the concentration of juvenile hormone (JH) to decrease in the aphid haemolymph., and particular form of parthenogenetic female called the sexuparae are produced. Sexuparae females produce sexual females and males that subsequently mate to produce overwintering eggs. Although parthenogenetic viviparous females and sexual oviparous females exhibit major differences in morphology and behavior, they share the same genome. This 
phenomenon, called reproductive polyphenism, is an example of aphid phenotypic plasticity [6].

The cellular and cytogenetic bases of reproductive polyphenism have been described for several aphid species $[7,8]$. In aphids, three generations are represented within one viviparous female: the mature embryos developing inside the maternal abdomen carry the first developmental stage of the third generation. This phenomenon is known as the 'telescoping of generations' [9]. The embryonic developments of asexual and sexual females are similar until the formation of undifferentiated germaria, which takes place at the end of germ band retraction (stage 18) [8]. The 32 germ cells within the germaria enter into early meiotic prophase (zygotene) and a partial association between bivalents occurs [7]. After this stage, the embryonic development and maturation of germ cells diverge between sexual and asexual females. Ancestral sexual development occurs under short photoperiod: the basal cells of germaria progress through meiosis; the sexual oocyte displays chromosome pairing, synapsis and meiotic recombinations. Meiosis is blocked in first meiotic prophase until the egg is fertilized and laid. Under long photoperiod, sexual oogenesis is inhibited [7]. The asexual oocyte ovulates in the posterior part of the germaria and forms a new follicle where it skips the first meiotic division and undergoes a single maturation division resulting in a discarded polar body and a diploid clonal oocyte. Synchronous mitotic divisions follow immediately without fertilization to initiate early embryogenesis, which continues within the parthenogenetic female abdomen and progresses through a series of 20 developmental stages [8].

We identified genes regulated in sexual and asexual aphid oogenesis by combining transcriptomic analyses and in situ hybridizations. In aphids, sexual and asexual reproduction can be analyzed in individuals possessing identical genomes. Sequencing of the LSR1 pea aphid genome [10] and the recent development of new genomic resources offer an unique opportunity to understand the molecular bases of sexual and asexual reproduction. Our results demonstrate that sexual and asexual reproduction pathways require unique and shared genetic programs.

\section{Results}

\section{Synchronization of sexual and asexual embryos}

The development of aphid embryos under long and short photoperiods is not synchronous. However, comparing the embryonic transcriptomes produced during sexual and asexual oogenesis requires synchronized embryo development. Thus, we used kinoprene (a JH analogue) to synchronize the development of sexual and asexual embryos under otherwise identical environmental conditions. Topical applications of kinoprene induces sexuparae reared under short photoperiods to produce asexual embryos instead of sexual embryos [11]. Compared to acetonetreatment (control), kinoprene treatment induced complete reversion of sexual to asexual reproduction in all larvae laid during the first two days after the onset of larviposition (Figure 1). Subsequent transcriptome comparisons were thus restricted to the five most-developed embryos dissected from synchronized kinoprene- or acetone-treated sexuparae. These embryos corresponded to the progeny laid on the first day.

\section{Differentially expressed transcripts}

Synchronized asexual (kinoprene-treated) and sexual (acetone-treated) embryos corresponding to embryonic developmental stages 18, 19 and 20, were collected from sexuparae $24 \mathrm{~h}, 48 \mathrm{~h}$ and $72 \mathrm{~h}$ after treatment, respectively. These are the final three developmental stages in aphid embryogenesis and correspond to eye differentiation (stage 18), muscle formation (stage 19) and the mature embryo (stage 20) [8]. We compared the transcriptomes of these embryos using microarrays. Pairwise correlations between the five biological replicates were high $(r>0.88)$, indicating good reproducibility. We compiled a list of 33 transcripts that were statistically differentially expressed: none of these transcripts were differentially regulated at developmental stage 18. Only one transcript (orb) was more abundant in asexual embryos than in sexual embryos at developmental stage 19, whereas 33 transcripts (including $o r b$ ) were differentially-regulated at developmental stage 20, including orb. At this final stage, four transcripts were more abundant in sexual embryos than in asexual embryos, with fold-change (FC) values between 1.49 and 2.86. Twenty-nine transcripts were more abundant in asexual embryos than in sexual embryos ( $F C$ values between 1.69 and 5.70) (Table 1).

Based on gene annotation in other organisms, 4 functional groups were defined (Table 1). Seven transcripts were related to oogenesis (e.g. nanos-1, orb, kelch); five transcripts were related to post-transcriptional regulation, such as mRNA polyadenylation (e.g. gld2) or mRNA silencing via RNA interference $(c b p 20)$; four transcripts were related to epigenetic regulation, such as histone modifications (suv4-20, uhrf1) or histone variants $(H 2 B .3, H 1)$; and three transcripts were assigned to cell cycle regulation (e.g. cyclin J, clasp1). Fourteen transcripts were not assigned to any functional category: among them, ten showed no similarity with characterized transcripts.

Our results displayed multiple genes related to oogesis (7/33). This oogenesis signature suggests that our experimental design, using artificially-induced asexual aphids, successfully targeted genes defining asexual or sexual oogenesis within the germaria or the first ovulated oocytes of the developing embryo. 

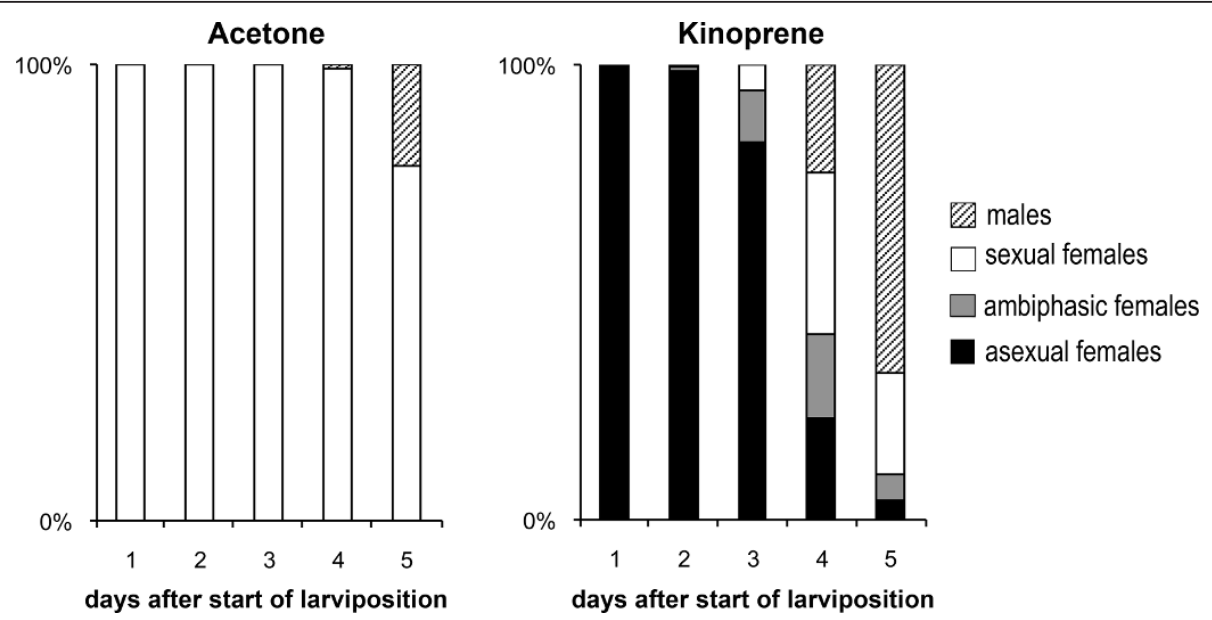

Figure 1 Effect of prenatal application of kinoprene on the type of embryonic ovaries. Acetone or kinoprene dissolved in acetone were topically applied to sexuparae individuals, $24 \mathrm{~h}$ after the fourth instar molt. Progeny batches were collected after initiation of larviposition, for five days every $24 \mathrm{~h}$. The reproductive phenotypes of the progeny were determined and the percentage of each morph (asexual females, ambiphasic females that contain a mixture of eggs and embryos, sexual females and males) is indicated. In control conditions (acetone), sexuparae sequentially produced sexual females and males: only sexual females were produced during the first three days after the onset of larviposition; males appeared the fourth day. Sexuparae treated with kinoprene produced exclusively parthenogenetic progeny the first day after the onset of larviposition. On day two, almost all the progeny were parthenogenetic. On day three, the majority of the progeny were still parthenogenetic. On days four and five, sexual morphs represented the majority of the progeny.

Localization of regulated transcripts in sexual and asexual ovaries

We compared the localization of these differentiallyexpressed transcripts in the ovaries of untreated asexual and sexual aphids using whole-mount in situ hybridization (WISH), a technique that is not suitable for transcript quantification. Specific antisense riboprobes were produced for 25 transcripts: five produced no signal (Additional file 1), four showed ubiquitous distribution (Additional file 2) and 16 transcripts were specifically expressed in germaria (containing germ and nurse cells) and/or in oocytes. These results on untreated aphids were consistent with the microarray data from acetoneor kinoprene-treated aphids: most of the candidate genes are expressed in the ovarioles. These 16 transcripts were grouped based on their patterns of expression in the two types of ovaries.

The first group included nine transcripts (bic $C$, histoneH1, gle1, gld2, pop2, arl6ip1, ACYPI007465, ACYPI010052 and ACYPI25088) that showed the same localization in sexual and asexual ovaries (Figure 2) despite microarray results that indicated higher levels of expression in asexual embryos. For five transcripts (gle1, bicC, ACYPI25088, ACYPI007465 and ACYPI010052), residual signals were observed in the developing embryo germ band (Figure 2).

The second group included two transcripts (uhrf1 and orb) that exhibited different localizations in the two types of ovaries. Uhrf1 signal was detected in germaria and oocytes in asexual ovaries, and a weak signal was detected in sexual oocytes (Figure 3). orb was detected in oocytes prior to ovulation (stage 0 ), in asexual germaria, and in unknown cellular structures located at the junction between sexual germaria and oocytes. Fluorescent detection of orb riboprobes was used together with fluorescent staining of actin filaments and DNA in order to define the intracellular localization of orb transcripts (Figure 4). In asexual ovaries, orb signals could be seen during early oogenesis, in stage 0 (oocyte formation) and in stage 1 (separation of the oocyte from the germarium) (Figure 4A). In sexual ovaries, the transcripts were detected only before ovulation at the junctions between the sexual germaria and the oocytes (Figure 4A). According to actin staining, a group of cells is localized at the junctions between sexual germaria and oocytes, these cells may correspond to the presumptive oocytes (Figure 4B). Thus, in asexual germaria, orb transcripts were detected in oocytes but not in presumptive oocytes; whereas in sexual ovaries, these transcripts were detectable only in cells that probably corresponded to presumptive oocytes but were not detectable in mature oocytes.

The third group included five transcripts ( $l s d 1$, lodestar, cyclin J, ACYPI39770 and ACYPI54656) that could be localized only in one type of ovary (Figure 5). These specific expression patterns were confirmed by microarray expression data for $l s d 1$, lodestar, cyclinJ and $A C Y$ P 39770. The $l s d 1$ riboprobe showed a strong 
Table 1 Description of the 33 differentially-expressed transcripts in sexual and asexual embryos

\begin{tabular}{|c|c|c|c|c|}
\hline Functional group & Gene identity & Similarity & Commentaries & $\begin{array}{c}\text { Fold } \\
\text { change }\end{array}$ \\
\hline \multirow[t]{7}{*}{ Oogenesis (7) } & ACYPI009492-RA & nudel & oocyte dorsal/ventral axis formation, egg activation & 3.86 \\
\hline & $\begin{array}{l}\text { ACYPI56634- } \\
\text { RA_0 }\end{array}$ & orb & $\begin{array}{l}\text { oocyte axis specification, mRNA polyadenylation, germ cell } \\
\text { development }\end{array}$ & 4.67 \\
\hline & ACYPI008811-RA & bicaudal-C & mitosis, oogenesis, ovarian follicle cell migration & 4.69 \\
\hline & ACYPI31336-RA & kelch & female germline ring canal formation, karyosome formation, oogenesis & 1.81 \\
\hline & Ap-nanos1 & nanos & $\begin{array}{l}\text { female meiosis chromosome segregation, germ cell development, } \\
\text { oogenesis }\end{array}$ & 3.69 \\
\hline & ACYPI23247-RA & putative kelch-like & female germline ring canal formation, karyosome formation, oogenesis & 1.69 \\
\hline & ACYPI46386-RA & $\begin{array}{l}\text { putative kelch-like } \\
\text { repeat }\end{array}$ & female germline ring canal formation, karyosome formation, oogenesis & -1.49 \\
\hline $\begin{array}{l}\text { Post- } \\
\text { transcriptionnal }\end{array}$ & ACYPI00753-RA & lodestar & termination of RNA polymerase II transcription, female meiosis & 3.39 \\
\hline \multirow[t]{4}{*}{ regulation (5) } & ACYPI003103-RA & Gld2 & $\begin{array}{l}\text { RNA polyadenylation, histone mRNA catabolic process, mRNA } \\
\text { processing }\end{array}$ & 4.03 \\
\hline & ACYPI50630-RA & Gle1 & poly $(A)+$ mRNA export from nucleus & 2.46 \\
\hline & ACYPI001842-RA & Pop2 & $\begin{array}{l}\text { RNA metabolic process, nuclear-transcribed mRNA poly(A) tail } \\
\text { shortening }\end{array}$ & 2.21 \\
\hline & ACYPI49482-RA & $\operatorname{cop} 20$ & $\begin{array}{l}\text { gene silencing by miRNA, production of siRNA involved in RNA } \\
\text { interference }\end{array}$ & 2.11 \\
\hline Epigenetic & $\begin{array}{l}\text { ACYPI002182- } \\
\text { RA }\end{array}$ & histone H2B.3 & chromatin assembly or disassembly, nucleosome assembly & 3.60 \\
\hline \multirow[t]{3}{*}{ regulation (4) } & $\begin{array}{l}\text { ACYPI005639- } \\
\text { RA }\end{array}$ & histone $\mathrm{H} 1$ & chromatin assembly or disassembly, nucleosome assembly & 2.85 \\
\hline & ACYPI28709-RA & Suv4-2OH1 & histone methylation, regulation of gene expression, epigenetic & 1.75 \\
\hline & $\begin{array}{l}\text { ACYPI007975- } \\
\text { RA }\end{array}$ & uhrfi & DNA repair, cell cycle, methylated histone residues binding & 2.48 \\
\hline \multirow[t]{3}{*}{ Cell cycle (3) } & ACYPI006224-RA & cyclin J & mitotic cell cycle, embryonic & 5.70 \\
\hline & ACYPI007770-RA & clasp1 & cell division, exit from mitosis, condensed chromosome kinetochore & 1.48 \\
\hline & ACYPI009671-RA & Drp-1 & cytokinesis, intracellular distribution of mitochondria & 1.79 \\
\hline \multirow[t]{4}{*}{ Other (4) } & ACYPI48893-RA & Arl6ip1 & cotranslational protein targeting to membrane & 4.25 \\
\hline & ACYPI48336-RA & Lsd-1 & lipid particle organization, regulation of lipid storage & -2.86 \\
\hline & ACYPI34416-RA & pol protein & RNA dependant DNA Polymerase/Reverse Transcriptase & -1.92 \\
\hline & ACYPI010082-RA & Six4 & fat body development, gonad development, mesoderm development & 1.56 \\
\hline \multirow[t]{10}{*}{ No similarity (10) } & ACYPI50301-RA & no similarity & & 3.92 \\
\hline & ACYPI39347-RA & hypothetical protein & & 2.70 \\
\hline & ACYPI38914-RA & predicted protein & & 2.04 \\
\hline & ACYPI54656-RA & hypothetical protein & & 2.12 \\
\hline & ACYPI25970-RA & hypothetical protein & & 3.86 \\
\hline & ACYPI010052-RA & hypothetical protein & & 3.78 \\
\hline & ACYPI007465-RA & hypothetical protein & & 2.53 \\
\hline & ACYPI25088-RA & hypothetical protein & & 2.20 \\
\hline & ACYPI39770-RA & no similarity & & 2.88 \\
\hline & $\begin{array}{l}\text { ACYPI005121- } \\
\text { RA }\end{array}$ & hypothetical protein & & -1.78 \\
\hline
\end{tabular}

Positive fold-change values indicate transcripts that were more abundant in asexual embryos. Negative fold-change values indicate transcripts that were more abundant in sexual embryos.

specific signal in the germaria and oocytes of sexual ovaries. In contrast, a specific signal for lodestar, cyclin J, ACYPI39770 and ACYPI54656 was detected only in asexual ovaries (Figure 5). lodestar transcripts were restricted to the diploid oocyte after separation from the germarium (stage 1). cyclin J, ACYPI39770 and ACYPI54656 transcripts were detected in asexual germaria and oocytes (Figure 5). In addition, ACYPI54656 and $A C Y P I 39770$ exhibited a residual signal in the germ bands of developing embryos (Figure 5). 


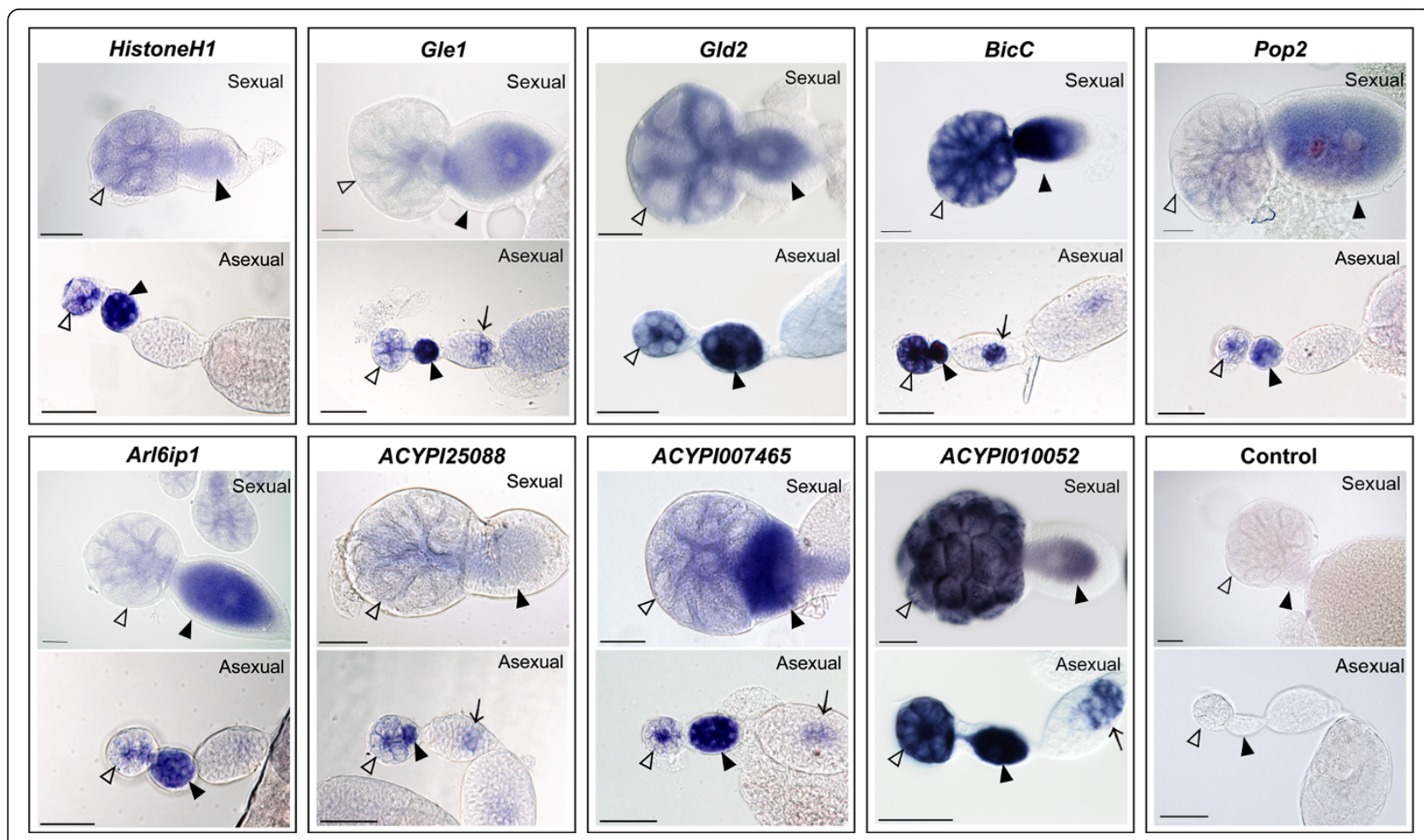

Figure 2 Transcripts with identical localizations in both sexual and asexual ovaries. Histone H1, gle1, gld2, bicC, pop2, arl6ip1, ACYPI25088, ACYPI007465 and ACYPl10052 transcripts were detected in germaria (hollow arrowheads) and oocytes (black arrowheads) of both sexual and parthenogenetic ovaries. Sense riboprobes were used as negative controls. For gle1, bicC, ACYPI25088, ACYPI007465 and ACYPI010052, a residual signal was observed in the developing embryo germ band (arrows). Bar scale: $50 \mu \mathrm{m}$.
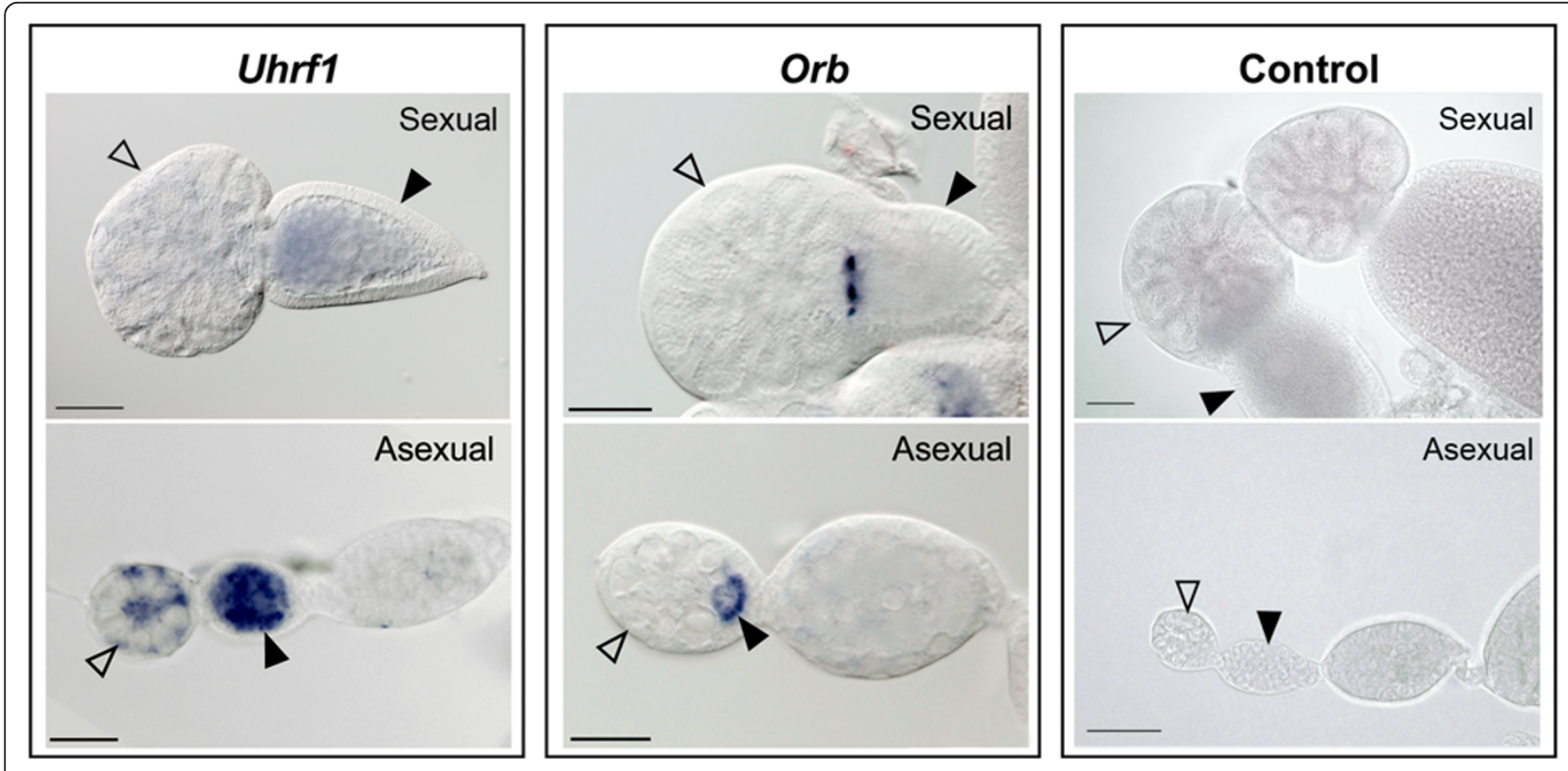

Figure 3 Transcripts with different localizations in sexual and asexual ovaries. Uhrf1 transcripts were detected in germaria (hollow arrowheads) and oocytes (black arrowheads) of asexual ovaries whereas a weak signal was detected in sexual oocytes (black arrowheads). Orb transcripts were detected in the basal parts of germaria in asexual and sexual ovaries (hollow arrowheads) with distinct localizations. In asexual germaria, these cells correspond to oocytes, whereas the cellular structures containing orb transcripts in sexual ovaries are undetermined. Sense riboprobes were used as negative controls. Bar scale: $50 \mu \mathrm{m}$. 


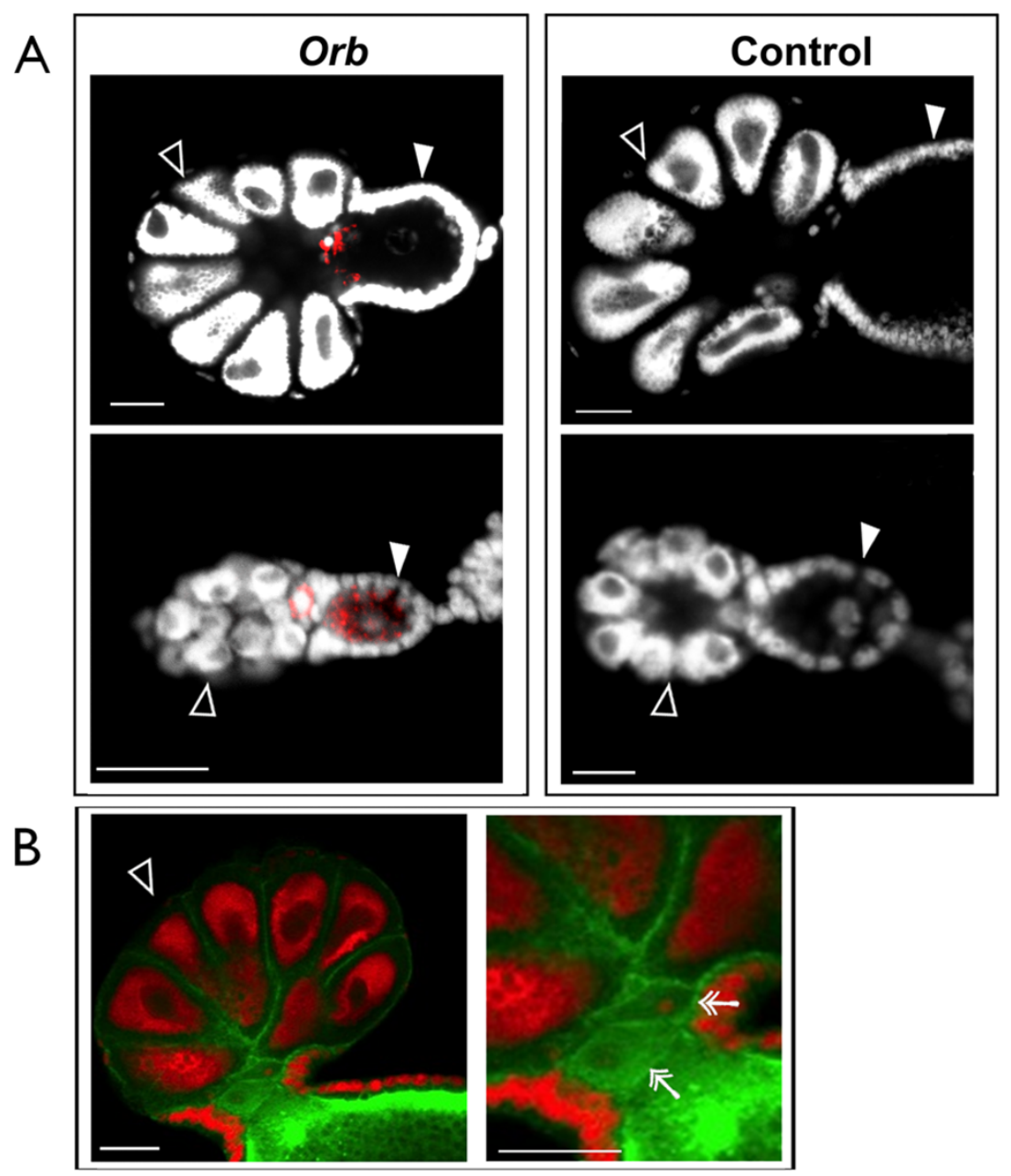

Figure 4 Detection of orb transcripts in sexual and asexual ovaries by fluorescent WISH. (A) orb riboprobes were detected with an antibody coupled to alexin fluorochrome (red) and DNA was stained with DAPI (white). In sexual ovaries (upper pictures), orb transcripts were detected at the basal part of the germaria (hollow arrowheads). In asexual ovaries (lower pictures), orb transcripts were detected in oocytes (white arrowheads) before ovulation (stage 0), and after ovulation (stage 1). (B) Actin filaments and DNA in sexual ovaries were stained with phalloidin (green) and propidium iodide (red), respectively. Cells at the basal part of the sexual germaria possibly correspond to presumptive sexual oocytes (double arrows). Orb sense riboprobes were used as negative controls. Bar scale: $30 \mu \mathrm{m}$.

\section{Discussion}

By coupling quantitative transcriptomic and qualitative WISH approaches, we identified 33 transcripts associated with the onset of divergent transcripts expression leading to the establishment of the sexual or asexual phenotype in aphids. The involvement of these candidate genes in determining the developmental direction of oogenesis is supported by: i) a strong signature of oogenesis among the regulated transcripts (7/33); and ii) the oocyte- and/or germarial-specific localizations of 16 of the transcripts. These data illustrate both shared and unique regulatory patterns of transcript expression for the two modes of reproduction.

First, we identified transcript expression profiles shared by sexual and asexual oogenesis. These transcripts are expressed in sexual and asexual germaria and/or oocytes but display differences in their expression levels and/or in their localization. In various organisms, gld2, bicC, pop 2 and orb are all involved in regulating the poly(A) tail length of maternal mRNAs. This process is a common mechanism of translational control essential for progression of meiosis and axis patterning during oocyte maturation [12-14]. The regulation of poly(A) tail length of maternal mRNAs results from a balance between concomitant deadenylation (translation repression) and polyadenylation (translation activation) [15]. In Drosophila melanogaster, gld2, bicC and orb have been described as part of the cytoplasmic polyadenylation complex that polyadenylates mRNAs and activates their translation $[13,16]$. Gld2-dependent 


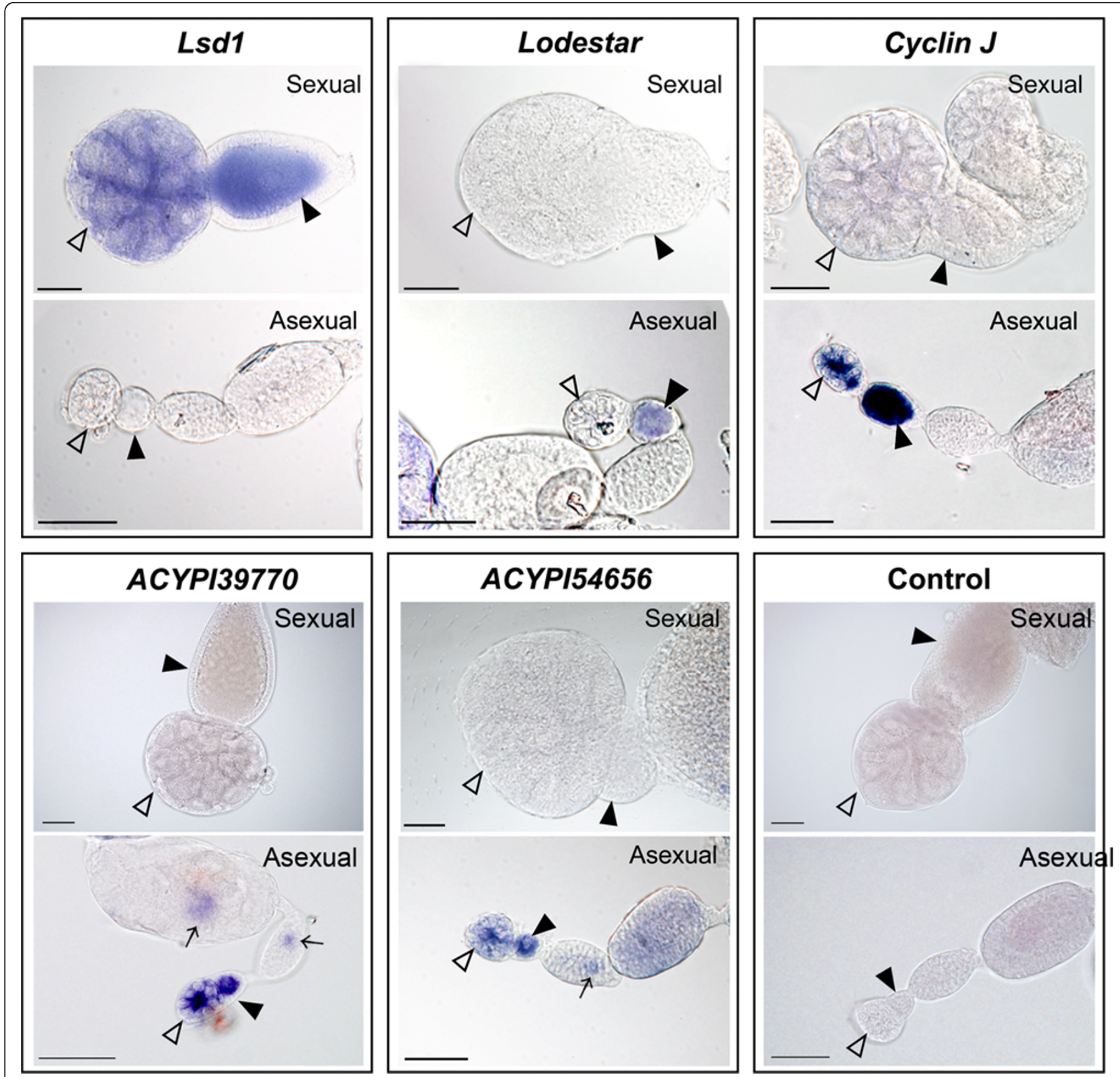

Figure 5 Transcripts detected specifically in one type of ovary. Lsd 1 transcripts were detected specifically in the germaria (hollow arrowheads) and haploid oocytes (black arrowheads) of sexual ovaries. lodestar, cyclin J, ACYPI39770, ACYPI54656 transcripts were detected specifically in asexual ovaries. Lodestar signal was detected specifically in asexual oocytes (black arrowheads). Cyclin J, ACYPI54656 and ACYPI39770 signals were detected in germaria (hollow arrowheads) and in oocytes (black arrowheads) of asexual ovaries. ACYPI54656 and ACYPI39770 showed a residual signal in the germ bands (arrows) of developing embryos. Sense riboprobes were used as negative controls. Bar scale: $50 \mu \mathrm{m}$.

mRNA polyadenylation and translational activation have been shown to be essential for oogenic meiosis [13]. On the other hand, mRNA deadenylation by the major deadenylation complex in Drosophila, CCR4-NOT, which includes the deadenylases CCR4, POP2, and four NOT proteins [17], promotes negative regulation of target mRNAs. Deadenylation of specific maternal mRNAs could be involved in the precise temporal and spatial mRNA localization necessary for axial patterning during oogenesis [12]. Expression of Api-gld2, Api-bicC, Apipop 2 and Api-orb in sexual and asexual germaria and/or in oocytes suggests that the balance between cytoplasmic polyadenylation and deadenylation in oocytes acts in the maturation of haploid as well as diploid oocytes in the pea aphid as it has been shown for other sexual organisms. Moreover, expression of the oocyte-specific linkers histoneH1 and uhrf1 in asexual and sexual oocytes suggests that epigenetic regulation is involved in 
pea aphid oogenesis. The linker histone $\mathrm{H} 1$ interacts with DNA to set-up and maintain the organization of the chromatin between the nucleosomes. Histone H1 proteins form a complex family of related proteins that have distinct species, tissue and developmental specificities and are involved in the epigenetic control of gene expression. The replacement of a somatic linker $H 1$ by an oocyte specific one (H1oo) during oogenesis has been described in a wide range of species (reviewed in $[18,19])$ and may promote a loosening in chromatin structure that appears to correlate with the initiation of meiosis [19]. The presence of Api-histoneH1 in germaria and oocytes suggests that this oocyte-specific variant of H1 might be involved in both sexual and asexual oogenesis in aphid. uhrf1 encodes an ubiquitin-like protein that is involved in two epigenetic silencing pathways through its regulation of DNA and histone methylation $[20,21]$. Although, uhrf1 has not been implicated in any oogenesis process to date, our results suggest a role for this protein in asexual and sexual oogenesis in aphids. Altogether, our results suggest that epigenetic regulatory mechanisms and the balance between cytoplasmic mRNA polyadenylation and deadenylation may have roles in regulating haploid and diploid oocyte maturation. Moreover, as orb transcript is the earliest to be differentially expressed during the developmental time course, we propose that Orb might play a major role in determining oocyte fate.

Second, we identified one gene, $l s d 1$, that is specifically expressed in pea aphid sexual germaria and oocytes. The $l s d 1$ transcript was not significantly detected either in asexual embryos according to microarray data (Additional file 3) or in asexual ovaries according to WISH. lsd 1 encodes a protein involved in the regulation of lipid droplet storage in eukaryotic cells [22]. Lipid droplets, like mature yolk bodies, are the major energy storage for oocytes and developing embryos in Drosophila [23]. Our results suggest that $l s d 1$ is required only for sexual oogenesis during which it may be involved in yolk accumulation. The absence of $l s d 1$ transcripts in asexual embryos is consistent with the absence of yolk in parthenogenetic oocytes [8]. Thus, identification of $l s d 1$ expression as a specific feature of sexual oogenesis validates the accuracy of our approach. Despite the existence of genes known to be involved in meiotic recombination in the pea aphid genome [24], we identified no such genes as specific markers of sexual oocytes. However, germline expression of meiotic genes such as spo11 was shown to be similar in sexual and asexual aphids. Expression of this gene seems to be modulated between asexual and sexual aphids by alternative splicing [25].

Finally, we identified three transcripts that were specific to asexual oogenesis. No significant transcription signal has been observed for these genes either in sexual embryos using microarrays (Additional file 3) or in sexual ovaries using WISH. That the polypeptide encoded by ACYPI39770 does not bear any similarity to a protein or protein domain of any other organism, suggests that ACYPI39770 is either an orphan gene or a gene with an unusual accelerated evolution rate. In Drosophila, cyclin $J$ and lodestar are involved in sexual oogenesis and early embryogenesis [26,27]. In aphids, their expression profiles suggest that they may haveroles in asexual oogenesis only. The absence of lodestar and cyclin $J$ transcripts from aphid sexual oocytes and embryos was particularly unexpected. Three lodestar paralogs were identified within the pea aphid genome (ACYPI000753, ACYPIO00590 and $A C Y P I 006971)$. Although the three copies were represented on the microarray by copy-specific oligonucleotides, only ACYPI000753 was differentially expressed in sexual and asexual embryos. Localization of ACYPI000753 transcripts by in situ hybridization showed that its expression was specific to asexual oocytes. However, although the sequence of the riboprobe used to locate ACYPI000753 transcripts was absent from the ACYPI006971 sequence, it shared $87 \%$ nucleotide identity with the unregulated paralog ACYPI000590. Therefore, cross hybridization between the $A C Y P I 000753$ riboprobe and $A C Y P I 000590$ transcripts cannot be excluded. Nevertheless, we detected no ACYPIO00753 expression in sexual oogenesis. This unexpected result might reflect the functional specialization of at least one of the lodestar paralogs in asexual oogenesis.

Among the 16 transcripts found to be specifically localized in germaria and/or oocytes, $l s d 1$ was the only one for which the expression level was found to be higher in sexual ovaries than in asexual ovaries according to the microarray results. This disproportion is consistent with the striking over-representation of transcripts that were upregulated in asexual embryos (29/33), as evidenced by the microarray analysis. Sexual oogenesis and asexual oogenesis display major inherent differences. Within embryos, the sexual oocyte nucleus remains blocked in meiosis prophase 1 whereas the asexual oocyte undergoes a modified but complete meiotic division immediately after ovulation [7]. This modified meiosis involves specific changes within the oocyte; self-organized asters are formed, that then recruit centriole precursors and pericentriolar material [28]. This process may be accompanied by the activation of microtubule stabilization factors or by the inactivation of microtubule destabilization factors specific to asexual oogenesis [28]. The predominance of genes that are upregulated in asexual embryos may be partially explained by the production of molecular components that are specifically required for asexual oogenesis but not for sexual oogenesis. 


\section{Conclusions}

In this study, we identified a number of putative embryonic target genes of the signaling cascade initiated by photoperiodic cues and transduced by JH. These genes are likely to be among those that direct sexual or asexual differentiation of the developing embryos. Our analysis of their expression patterns represents the first time the molecular bases of aphid sexual and asexual oogenesis have been addressed. Altogether, our results showed that aphid asexual oogenesis requires the same genes as sexual oogenesis but that these two distinctive differentiation processes display specific gene expression programs.

\section{Methods}

\section{Aphid rearing, dissection and extraction}

Clonal descendants from the sequenced Acyrthosiphon pisum LSR1 clone were reared on Vicia fabae at $18^{\circ} \mathrm{C}$ and at low density (5 individuals per plant) to prevent the production of winged morphs. Parthenogenesis was maintained under long photoperiod (16 h). In our conditions, the shortening of the photoperiod (12 h) requires three generations to induce the production of sexual morphs. The first asexual generation was reared under long photoperiod and transferred to short photoperiod $(12 \mathrm{~h})$ after the third instar moult. Adult asexual virginoparae females produced the second asexual generation, named sexuparae. Adult sexuparae, reared in short photoperiods, produced the third generation of sexual females oviparous, males, and asexual viviparous females.

\section{Kinoprene application}

Aphid sexuparae were synchronised at the fourth instar moult, during a 6 hour window. $24 \mathrm{~h}$ after, $400 \mathrm{ng}$ of kinoprene (Sigma Aldrich) diluted in $50 \mu \mathrm{L}$ of acetone were ectopically applied on sexuparae abdomens of 100 females. The progeny of treated aphids was collected daily for 5 days. When they reached adulthood, collected aphids were dissected and their reproductive type was determined according to the type of ovaries they contained within their abdomen: sexual ovaries that are only made of haploid eggs, ambiphasic ovaries that contain a mixture of eggs and embryos, and asexual ovaries that are only constituted of embryos at different stages of development. Ectopic applications of $50 \mu \mathrm{L}$ of acetone were performed as a negative control.

\section{Sampling, RNA isolation and microarray hybridization}

Synchronized sexuparae were randomly divided into 2 batches and treated with kinoprene or acetone (as a control) $24 \mathrm{~h}$ after fourth instar moult (Additional file 4). 25 treated sexuparae were collected 24,48 , and 72 hours after kinoprene or acetone application. For each condition, the 5 most developed embryos were isolated from each of the 25 treated sexuparae by dissection, pooled together, frozen into liquid nitrogen and stored at $-80^{\circ} \mathrm{C}$. These most developed embryos within treated sexuparae collected 24,48 , and 72 hours after treatment correspond to the developemental stage 18,19 or 20 respectively. This procedure was repeated 5 times to generate as much independent biological replicates. Total RNAs were isolated from each sample by using the RNeasy Mini kit (Qiagen) according to manufacturer's instructions. RNA quality was checked on Bioanalyser (Agilent) and quantified on Nanodrop (Thermo scientific). For each sample, $20 \mu \mathrm{g}$ of total RNAs were sent to the NimbleGen expression array platform (Roche). Double stranded cDNA synthesis and $\mathrm{Cy} 3$ end-labelling were performed by NimbleGen.

\section{Microarrays design and analysis}

Custom microarrays were constructed on NimbleGen (Roche) $385 \mathrm{~K} 4$-plex ( $4 \times 72,000$ probes). 24,011 transcripts were represented by 360 -mers oligonucleotides probes (MIAMExpress, http://www.ebi.ac.uk/miamexpress/, microarray: INRA-BF2I_A.pisum_NimblegenACYPI_4x72k_v1; Array express accession: A-MEXP1999). Hybridization and scanning were performed by NimbleGen, providing the final raw data file (experiment: Aphid_embryo, ArrayExpress accession: E-MEXP3481). The limma 2.16 package [29] in R 2.9.2 [30] was used for statistical analyses. For each transcript the average of the 3 probe signals was considered. Median normalization between microarrays was performed. A design matrix incorporating effects of treatment $\mathrm{K}$ (kinoprene) or A (acetone) and developing stage (18, 19, 20) was constructed. Variance was adjusted for Bayesian fitting of the model. Differential expression was determined with a two-way ANOVA considering the effect of time and treatment between 3 contrasts: 'A18 versus K18', 'A19 versus K19' and 'A20 versus K20'. Significance of differential expression was assigned with a $10 \%$ false discovery rate (FDR) [31]. Annotation of differentially expressed transcripts was curated using pea aphid RNAseq data (published in SRA of NCBI), the software CAP3 [32] and Blastx on NCBI non-redundant protein sequence data bank. Functional annotations were performed using the Gene Ontology (http://www.geneontology.org/).

\section{Riboprobe synthesis for in situ hybridization}

Templates for synthesis of riboprobes were obtained from full-length cDNAs collection (ACYPI003103, ACYPI010052 [33]) or amplified by RT-PCR and cloned (Additional file 5). Total RNAs were extracted from parthenogenetic virginoparae females with RNeasy plant kit (Qiagen). DNA contaminations were removed by a 
treatment with RQ1 RNase-free DNAse (Promega). First strand cDNAs were produced from $1 \mu \mathrm{g}$ of total RNAs by using random primer 9 (New England BioLabs) and SuperScript ${ }^{\circledR}$ III Reverse Transcriptase (Invitrogen) following the supplier's instructions. cDNAs were used as a matrix for PCR amplification with specific primers (Additional file 5). Amplified fragments were cloned into the StrataClone PCR Cloning Vector pSC-A-amp/ kan (StrataClone) or pENTR Directional TOPO (Invitrogen) and sequenced (Genoscreen). Linear PCR products were amplified from cloned sequence with universal primers M13 and used as a matrix for synthesis of sense and antisense riboprobes by using digoxigenin-labelled dNTPs and the appropriate RNA polymerase (T3/T7/ SP6) supplied in the DIG RNA labelling kit (Roche). Remaining DNA was removed with RQ1 RNase-free DNAse treatment (Promega) and riboprobes were purified with the RNeasy mini kit (Qiagen). Riboprobe quality and quantity were checked on Nanodrop (Thermo scientific).

\section{Whole mount in situ hybridization and microscopy}

Whole mount in situ hybridization was performed on ovaries of sexual or asexual nymphs. Ovaries were dissected and fixed in $4 \%$ paraformaldehyde in $1 \times$ PBS at room temperature (RT) for $30 \mathrm{~min}$. Ovaries were washed in $50 \%$ methanol for $30 \mathrm{~min}$, then dehydrated and stored in methanol at $-20^{\circ} \mathrm{C}$. They were rehydrated in graded methanol/PTw ( $1 \times$ PBS, $0.2 \%$ Tween-20) solutions $(70 \% ; 50 \% ; 30 \%, 10 \mathrm{~min}$ each), post fixated in $4 \%$ paraformaldehyde in $1 \times$ PBS for $20 \mathrm{~min}$ and washed 3 times for $5 \mathrm{~min}$ in PTw. Ovaries were washed for 45 min in $1 \%$ SDS, $0.5 \%$ Tween-20, $50 \mathrm{mM}$ Tris- $\mathrm{HCl}(\mathrm{pH}$ 7,5), $1 \mathrm{mM}$ EDTA ( $\mathrm{pH}$ 8), $150 \mathrm{mM} \mathrm{NaCl}$ and 5 times for 5 min with PTw. Pre-hybridization was performed $10 \mathrm{~min}$ in hyb-wash solution $(5 \times \mathrm{SSC}, 50 \%$ formamide, $0.1 \%$ Tween-20) and 1 hour at $65^{\circ} \mathrm{C}$ in hyb solution (0.3\% SDS, $5 \times$ SSC, $50 \%$ formamide, $100 \mu \mathrm{g} / \mathrm{mL}$ heparin, $0.1 \%$ Tween $-20,100 \mu \mathrm{g} / \mathrm{mL}$ yeast RNA, $10 \mathrm{mM}$ DTT). Hybridization was performed overnight at $65^{\circ} \mathrm{C}$ with 500 $\mathrm{ng} / \mathrm{mL}$ of denatured sense or antisense RNA probe diluted in hyb solution. Nonspecific hybridizations were washed off twice at $65^{\circ} \mathrm{C}$ in hyb-wash solution for 30 min, 3 times in 50\% hyb-wash solution/PTw $30 \mathrm{~min}$ and 3 times in PTw for $10 \mathrm{~min}$ at RT. Ovaries were incubated in blocking solution (0.2\% BSA in PTw) for $5 \mathrm{~min}$ and $1 \mathrm{~h}$ at RT, and bound digoxigenin labelled probes were detected overnight at $4^{\circ} \mathrm{C}$ with anti-DIG-alkaline phosphatase (AP) Fab fragments (Roche) diluted 1:2000 in blocking solution. Ovaries were washed 4 times 20 min and twice for $1 \mathrm{~h}$ in blocking solution at RT. After

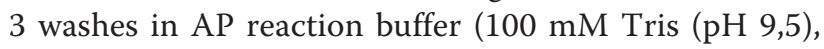
$100 \mathrm{mM} \mathrm{NaCl}, 5 \mathrm{mM} \mathrm{MgCl} 2,0.2 \%$ Tween-20), signal was revealed with $4 \mu \mathrm{l}$ NitroBlue Tetrazolium/5-Bromo-
4-Chloro-3-Indolyl Phosphate (NBT/BCIP) Stock Solution (Roche)/ml AP reaction buffer. Finally, ovaries were rinsed at least 3 times for $5 \mathrm{~min}$ in PTw, dehydrated 5 min in methanol, and rinsed twice again $5 \mathrm{~min}$ in PTw, before mounting in $70 \%$ glycerol in PBS. Samples were photographed with a microscope Nikon 90i connected to a Nikon type DS-Ri1 camera or with Olympus BX61 connected to Nikon DS-Fil camera.

For the fluorescence protocol, the same procedure was followed until hybridization step. Then TSATM Biotin System (Perkin Elmer, Waltham, USA) was used for detection as follows. After overnight hybridization of the probes, nonspecific probes were washed off twice at $65^{\circ} \mathrm{C}$ in hyb-wash solution for $30 \mathrm{~min}$, once in $50 \%$ hyb-wash solution/TNT buffer $(0.1 \mathrm{M}$ Tris- $\mathrm{HCl}(\mathrm{pH}$ 7.5), $0.15 \mathrm{M} \mathrm{NaCl}, 0.05 \%$ Tween-20) for $30 \mathrm{~min}$ at RT, and 3 times in TNT buffer for $10 \mathrm{~min}$ at RT. Ovaries were blocked with the TSA ${ }^{\mathrm{TM}}$ blocking reagent, for 30 min at RT. Bound digoxigenin labelled probes were detected with HRP conjugated anti-DIG (BoehringerMannheim) (1:250) diluted in TNT buffer at $4^{\circ} \mathrm{C}$ for overnight incubation. Ovaries were washed four times in TNT buffer for $15 \mathrm{~min}$. The TSA TM Biotin System Amplification was used by incubation 20 min into a diluted solution of the Biotinyl Tyramide (Amplification Reagent) in 1× Amplification Diluent (1:50). HRP catalyzes the formation of TSA free radicals, which form covalent bounds to tyrosine residues proximal to HRP. They were then washed 3 times 15 min in TNT buffer, and 4 times $20 \mathrm{~min}$ in PTw. Ovaries were incubated $2 \mathrm{~h}$ into Alexa 594-conjugated Streptavidin (Invitrogen) diluted in TNT buffer (1:500) and rinsed into PTw before performing a nuclear stain with TO$\mathrm{PRO}^{\circledR}{ }^{\circledR}$ (1:1000 in PTw) for $30 \mathrm{~min}$. Finally, ovaries were washed 3 times in PTw before mounting. Images were acquired and processed using an Olympus FV1000 confocal microscope.

\section{Phalloidin and propidium iodide staining}

Oviparous ovaries were dissected and fixed as previously described. Samples were washed 3 times in PTw, followed by PTw for $30 \mathrm{~min}$ and 2\% Normal Goat Serum (NGS)/BPTw for $60 \mathrm{~min}$. Samples were then incubated overnight at $4^{\circ} \mathrm{C}$ with Alexa Fluor 488 phalloidin (Invitrogen) at a concentration of 1:200 to visualize F-actin. The next day samples were washed 4 times in PTw, and placed in $20 \mu \mathrm{g} / \mathrm{mL}$ RNaseA (QIAGEN)/PTw for $3 \mathrm{~h}$ at RT. Samples were washed twice in PTw, and incubated with $10 \mu \mathrm{g} / \mathrm{mL}$ propidium iodide (Invitrogen) in $20 \mu \mathrm{g} /$ $\mathrm{mL}$ RNaseA/PTw for $60 \mathrm{~min}$ at RT to visualize DNA. Samples were washed 4 times in PTw and mounted in Vectashield (Vector Laboratories). Images were acquired and processed using an Olympus FV1000 confocal microscope. 


\section{Author's information}

AG, SJP and DT: INRA, UMR 1099 BiO3P, Biologie des Organismes et des Populations appliquée à la Protection des Plantes, 35653 Le Rheu cedex, France

SS and TH: Okazaki Institute for Integrative Bioscience, National Institute for Basic Biology, National Institutes of Natural Sciences, Higashiyama, Myodaiji, Okazaki, 444-8787, Japan

\section{Additional material}

Additional file 1: Transcription of nudel, drp1, clasp1, cbp1 and ACYPI005121 in parthenogenetic ovaries. nudel, drp1, claspl, cbpl and ACYPI005121 transcripts localizations were investigated by in situ hybridization on parthenogenetic ovaries including germaria (hollow arrowheads) and in oocytes (black arrowheads). Specific antisense riboprobes gave no signal for these transcripts. Sense riboprobes were used as negative controls. Bar scale: $50 \mu \mathrm{m}$.

Additional file 2: Transcription of six4, suv4-20, kelch and ACYPI38914 in parthenogenetic ovaries. six4, suv4-20, kelch and ACYPI38914 transcripts localizations were investigated by in situ hybridization on parthenogenetic ovaries including germaria (hollow arrowheads) and in oocytes (black arrowheads). Specific antisense riboprobes showed ubiquitous distribution of these transcripts. Sense riboprobes were used as negative controls. Bar scale: $50 \mu \mathrm{m}$.

Additional file 3: Quantitative expression of Isd1, lodestar, cyclin J and ACYPI39770 in sexual and asexual embryos measured by microarrays. Transcription levels of Isd-1, lodestar, cyclin-J and ACYPI39770 were measured by microarrays in sexual (full line) and asexual (dot line) embryos. Log 2 of expression value was provided for each developmental stage $(18,19$ and 20$)$. The grey area contained the values comprised between the first and third quartile calculated for the 24011 transcripts included in the microarray. Standard errors were measured for the 5 biological replicates.

\section{Additional file 4: Experimental design to synchronize the} development of pea aphid sexual and asexual embryos. Sexuparae were synchronized at the fourth instar moult in a six hours window. Synchronous sexuparae were randomly separated into two batches; one treated with acetone (A) and one kinoprene (K) $24 \mathrm{~h}$ after fourth instar moult. Within each batch, sexuparae were collected $24 \mathrm{~h}, 48 \mathrm{~h}$ and $72 \mathrm{~h}$ after treatment, dissected and the 5 most developed embryos were collected for further RNA extraction.

Additional file 5: Primer sequences for cDNA amplification and riboprobe synthesis. Specific PCR primers were designed for each of the regulated transcripts in order to amplify cDNA.

\section{Acknowledgements}

Hubert Charles, Stefano Colella (INRA INSA UMR BF2I, France) and Grégory Guernec (INRA UMR SCRIBE, France) are acknowledged for microarrays design and analyses. We acknowledge Sylvie Hudaverdian (INRA UMR BiO3P, France), Chantal Cauty and Jérôme Burgeon (INRA UMR SCRIBE, France) for technical support. Jim Hardie (Imperial College of London, UK) provided advices on the use of JH analogs. Owain Edwards (CSIRO, Australia), JeanRené Huynh (CNRS Institut Curie, France), Luc Paillard (CNRS, UMR 6061, France), Gaël Le Trionnaire and Jean-Christophe Simon (INRA UMR BiO3P, France) provided valuable comments on the manuscript. This work was funded by ANR programs 'Aphicible' and 'Holocentrisme', by the Japan Society for the Promotion of Sciences and by french "Ministère de l'enseignement supérieur et de la recherche".

\section{Author details}

'INRA, UMR 1349 IGEPP, Institut de Génétique Environnement et Protection des Plantes, 35653 Le Rheu cedex, France. ${ }^{2}$ Okazaki Institute for Integrative
Bioscience, National Institute for Basic Biology, National Institutes of Natural Sciences, Higashiyama, Myodaiji, Okazaki, 444-8787, Japan.

\section{Authors' contributions}

$A G, S S, S J P$ and DT designed the research. AG and TH performed the experiments. AG, SS, SJP and DT analyzed data. AG, SJP and DT wrote the manuscript. All the authors read and approved the final manuscript.

Received: 21 September 2011 Accepted: 15 February 2012 Published: 15 February 2012

\section{References}

1. Lost sex, the evolutionary biology of parthenogenesis. Edited by: Schön I, Martens K, van Dijk P. Springer; 2009:

2. Simon JC, Delmotte F, Rispe C, Crease T: Phylogenetic relationships between parthenogens and their sexual relatives: the possible routes to parthenogenesis in animals. Biol J Linn Soc 2003, 79:151-163.

3. Schwander T, Crespi BJ: Multiple direct transitions from sexual reproduction to apomistic parthenogenesis in tinema stick insects. Evolution 2009, 63:84-103.

4. Hörandl E: Geographical Parthenogenesis: Opportunities for Asexuality. In Lost sex, the evolutionary biology of parthenogenesis. Edited by: Schön I, Martens, K., and van Dijk, P. Springer; 2009:161-186.

5. Dixon AFG: Aphid ecology. 2 edition. Chapman \& Hall; 1998

6. Le Trionnaire G, Hardie J, Jaubert-Possamai S, Simon JC, Tagu D: Shifting from clonal to sexual reproduction in aphids: physiological and developmental aspects. Biol Cell 2008, 100:441-451.

7. Blackman RL: Reproduction, cytogenetics and development. In Aphids: their biology, natural enemies and control. Volume 2A. Edited by: Minks AKaH, A.P. Amsterdam: Elsevier; 1987:163-195.

8. Miura T, Braendle C, Shingleton A, Sisk G, Kambhampati S, Stern DL: A comparison of parthenogenetic and sexual embryogenesis of the pea aphid Acyrthosiphon pisum (Hemiptera: Aphidoidea). J Exp Zool Part B 2003, 295B:59-81.

9. Stevens NM: A study of the germ cells of Aphis rosae and Aphis oenotherae. Journal Experimental Zoology 1904, 313-333.

10. International Aphid Genomics Consortium: Genome sequence of the pea aphid Acyrthosiphon pisum. PLoS Biol 2010, 8:e1000313.

11. Corbitt TS, Hardie J: Juvenile-hormone effects on polymorphism in the pea aphid, Acyrthosiphon pisum. Entomol Exp Appl 1985, 38:131-135.

12. Chicoine J, Benoit P, Gamberi C, Paliouras M, Simonelig M, Lasko P: Bicaudal-C recruits CCR4-NOT deadenylase to target mRNAs and regulates oogenesis, cytoskeletal organization, and its own expression. Dev Cell 2007, 13:691-704.

13. Benoit P, Papin C, Kwak JE, Wickens M, Simonelig M: PAP- and GLD-2-type poly(A) polymerases are required sequentially in cytoplasmic polyadenylation and oogenesis in Drosophila. Development 2008, 135:1969-1979.

14. Eckmann CR, Crittenden SL, Suh N, Kimble J: GLD-3 and control of the mitosis/meiosis decision in the germline of Caenorhabditis elegans. Genetics 2004, 168:147-160

15. Kim JH, Richter JD: Opposing polymerase-deadenylase activities regulate cytoplasmic polyadenylation. Mol Cell 2006, 24:173-183.

16. Castagnetti S, Ephrussi A: Orb and a long poly(A) tall are required for efficient oskar translation at the posterior pole of the Drosophila oocyte. Development 2003, 130:835-843

17. Temme C, Zhang LB, Kremmer E, Ihling C, Chartier A, Sinz A, Simonelig M, Wahle E: Subunits of the Drosophila CCR4-NOT complex and their roles in mRNA deadenylation. RNA-Publ RNA Soc 2010, 16:1356-1370.

18. Izzo A, Kamieniarz K, Schneider R: The histone $\mathrm{H} 1$ family: specific members, specific functions? Biol Chem 2008, 389:333-343.

19. Godde JS, Ura K: Dynamic alterations of linker histone variants during development. Int J Dev Biol 2009, 53:215-229.

20. Qin WH, Leonhardt H, Spada F: Usp7 and Uhrf1 Control Ubiquitination and Stability of the Maintenance DNA Methyltransferase Dnmt1. J Cell Biochem 2011, 112:439-444

21. Hashimoto H, Horton JR, Zhang X, Cheng XD: UHRF1, a modular multi-domain protein, regulates replication-coupled crosstalk between DNA methylation and histone modifications. Epigenetics 2009, 4:8-14. 
22. Beller M, Bulankina AV, Hsiao HH, Urlaub $H$, Jackle $H$, Kuhnlein RP: PERILIPIN-Dependent Control of Lipid Droplet Structure and Fat Storage in Drosophila. Cell Metab 2010, 12:521-532.

23. Cermelli S, Guo Y, Gross SP, Welte MA: The lipid-droplet proteome reveals that droplets are a protein-storage depot. Curr Biol 2006, 16:1783-1795.

24. Srinivasan DG, Fenton B, Jaubert-Possamai S, Jaouannet M: Analysis of meiosis and cell cycle genes of the facultatively asexual pea aphid, Acyrthosiphon pisum (Hemiptera: Aphididae). Insect Mol Biol 2010, 19:229-239.

25. Srinivasan D, Año L, Fenton B, Jaubert-Possamai S, Stern DL: Molecular mechanism of facultative parthenogenesis in the pea aphid,

Acyrthosiphon pisum. 2009, 8th International symposium on aphids; 8-12 June 2009; Catania (Italy).

26. Kolonin MG, Finley RL: A role for cyclin $\mathrm{J}$ in the rapid nuclear division cycles of early Drosophila embryogenesis. Dev Biol 2000, 227:661-672.

27. Szalontai T, Gaspar I, Belecz I, Kerekes I, Erdelyi M, Boros I, Szabad J: Horka (D), a Chromosome Instability-Causing Mutation in Drosophila, Is a Dominant-Negative Allele of lodestar. Genetics 2009, 181:367-377.

28. Riparbelli MG, Tagu D, Bonhomme J, Callaini G: Aster self-organization at meiosis: a conserved mechanism in insect parthenogenesis? Dev Biol 2005, 278:220-230

29. Smyth GK: Linear models and empirical Bayes methods for assessing differential expression in microarray experiments. Statistical Applications in Genetics and Molecular Biology 2004, 1-26.

30. R Development Core Team: $R$ : a language and environment for statistical computing Vienna, Austria: R foundation for statistical computing; 2009.

31. Benjamini $Y$, Hochberg $Y$ : Controlling the false discovery rate - A practical and powerful approach ot multiple testing. J R Stat Soc Ser B-Methodol 1995, 57:289-300.

32. Huang XQ, Madan A: CAP3: A DNA sequence assembly program. Genome Res 1999, 9:868-877.

33. Shigenobu S, Richards $S$, Cree AG, Morioka M, Fukatsu T, Kudo T, Miyagishima S, Gibbs RA, Stern DL, Nakabachi A: A full-length cDNA resource for the pea aphid, Acyrthosiphon pisum. Insect Mol Biol 2010, 19:23-31.

doi:10.1186/1471-2164-13-76

Cite this article as: Gallot et al: Sexual and asexual oogenesis require the expression of unique and shared sets of genes in the insect Acyrthosiphon pisum. BMC Genomics 2012 13:76.

\section{Submit your next manuscript to BioMed Central and take full advantage of:}

- Convenient online submission

- Thorough peer review

- No space constraints or color figure charges

- Immediate publication on acceptance

- Inclusion in PubMed, CAS, Scopus and Google Scholar

- Research which is freely available for redistribution

Submit your manuscript at www.biomedcentral.com/submit
Biomed Central 